\section{Insights into pregnancy associated and atypical hemolytic uremic syndrome}

Obstetric Medicine 2018, Vol. II(3) 137-140

(C) The Author(s) 2018

Reprints and permissions: sagepub.co.uk/journalsPermissions.nav DOI: $10.1177 / 1753495 \times 18780099$ journals.sagepub.com/home/obm

(9SAGE

\author{
Sophie Grand'Maison ${ }^{1,2}$ and Stephen Lapinsky ${ }^{3}$
}

Received 12 March 2018; accepted 7 May 2018

\section{Introduction}

Hemolytic uremic syndrome (HUS) is defined by microangiopathic hemolytic anemia, renal dysfunction, and thrombocytopenia. ${ }^{1}$ It is the result of an insult to endothelial cells that may be caused by different mechanisms including Shiga toxin-induced HUS, autoimmune diseases, drugs, infections, and pregnancy (p-HUS). ${ }^{2}$ Atypical HUS (aHUS), unlike HUS, is associated with genetic or acquired dysfunction of the alternative complement pathway. ${ }^{3}$ Pregnancy and postpartum are recognized as specific triggers of aHUS, and this association led to the now recognized entity of pregnancyassociated aHUS (p-aHUS). ${ }^{4}$ p-aHUS is a rare and life-threatening disease that affects approximately 1 in 25,000 pregnancies. ${ }^{5}$ There is no specific test and the diagnosis of this syndrome may be challenging, as the clinical characteristics are very similar to severe preeclampsia and hemolysis elevated liver enzymes and low platelet syndrome (HELLP). In the past, plasmapheresis and fresh frozen plasma (FFP) were the two most reported treatments. However, more recently, eculizumab, a humanized monocolonal antibody to complement component five (C5), has been used. ${ }^{6}$ In 2017, three cohort studies were published which we review below (Table 1). They provide valuable data to understand the presentation, outcomes and other specific characteristics of p-aHUS.

\section{Pregnancy-associated hemolytic uremic syndrome and complement gene variants}

A study of an international cohort of patients aimed to identify presentation, outcomes, and frequency of complement alternative pathway gene variants in women with p-HUS. ${ }^{2}$ This was a retrospective (between 1983 and 2013), multicenter study including patients from France, United Kingdom and Italy identified through computerized databases. They included 87 women (mean age $29 \pm 6$ years) with a diagnosis of p-HUS for whom complement workup had been done. p-HUS was defined as HUS that presented during pregnancy or up to 12 weeks postpartum. HUS was defined by the association of at least three criteria including hemolytic anemia, thrombocytopenia, acute kidney insufficiency (AKI), or features of thrombotic microangiopathy (TMA) at kidney biopsy. Patients with preeclampsia diagnosed before the development of TMA, HELLP syndrome, massive postpartum bleeding, or other identified causes of secondary HUS (lupus, antiphospholipid syndrome) were excluded. Complement gene variants studies were done according to each center.

Familial history of aHUS was present in $16 \%(14 / 87)$ cases and personal history in $8 \%(\mathrm{n}=7 / 87)$, all unrelated to pregnancy. The majority of cases $(\mathrm{n}=63,76 \%)$ presented in the postpartum period (mean $14 \pm 12$ days). Twenty patients (24\%) developed p-HUS during pregnancy, mostly (18/20) in the third trimester. All women had measurement of ADAMTS13 (a disintegrin and metalloproteinase with a thrombospondin type 1 motif, member 13) and activity was $>10 \%$ in all cases (as expected by definition). AKI was severe in $71 \%(56 / 78)$ of cases needing hemodialysis at presentation. First-line therapy was plasmapheresis in $78 \%(56 / 72)$ of cases, and after 2011, eculizumab was given to four patients as a second-line therapy after delivery. There were no maternal deaths, but they recorded $14 \%(n=11)$ fetal or neonatal deaths. At the end of the follow-up period ( $7.2 \pm 5.2$ years), $28 \%(22 / 78)$ of patients had a glomerular filtration rate $>60 \mathrm{ml} / \mathrm{min}, 19 \%(15 / 78)$ had chronic kidney disease, $53 \%(41 / 78)$ progressed to end-stage renal disease (ESRD), 27\% (24/87) had a kidney transplant and 21\% (18/87) had a recurrence of HUS. Among the four patients who received eculizumab, three of them had a complete recovery but the other one remained dialysis dependent.

The complement component assays showed a reduction of $\mathrm{C} 3$ in $39 \%(29 / 74)$ of women, in whom complement gene variants were found in $79 \%(24 / 29)$. Novel or rare variants of complement genes were present in 56\% (49/87) of women and $9 \%(8 / 87)$ had combined variants. Women with detected variants had worse outcomes than the others: need for hemodialysis ( $81 \%$ vs. $58 \%, \mathrm{p}=0.02)$, relapses $(38 \%$ vs. $16 \%, \mathrm{p}=0.04)$, and progression to ESRD (64\% vs. $36 \%$, $\mathrm{p}=0.01$ ). There was no difference in the gene variant distributions between women who had p-HUS during pregnancy or in the postpartum period. The complement gene variants found in p-HUS were similar to those related to aHUS found in the three national registries. Renal prognosis was poor with plasma exchange and plasma infusion treatment, and was similar if p-HUS presented during or after pregnancy.

The authors conclude that women with p-HUS should be considered as having aHUS triggered by pregnancy (p-aHUS) and should receive eculizimab as first-line therapy, as the renal prognosis is poor without efficacious treatment.

This retrospective cohort included a large number of women with p-aHUS based on complement evaluation and genetic analysis. It establishes the link between p-HUS and p-aHUS. This study combined three registries from three different countries and all records were reviewed, which reduces potential selection bias. However, little

'Medicine Department, Centre Hospitalier de l'Université de Montréal, Montréal, Canada

${ }^{2}$ Centre de Recherche du Centre Hospitalier de l'Université de Montréal, Montréal, Canada

${ }^{3}$ Interdepartmental Division of Critical Care Medicine, University of Toronto, Toronto, Canada

\section{Corresponding author:}

Sophie Grand'Maison, 1000 St-Denis, Montreal, QC, H2X 0CI, Canada. Email: sophie.grandmaison@umontreal.ca 
Table I. Comparison between the three cohort studies of hemolytic-uremic syndrome in pregnancy.

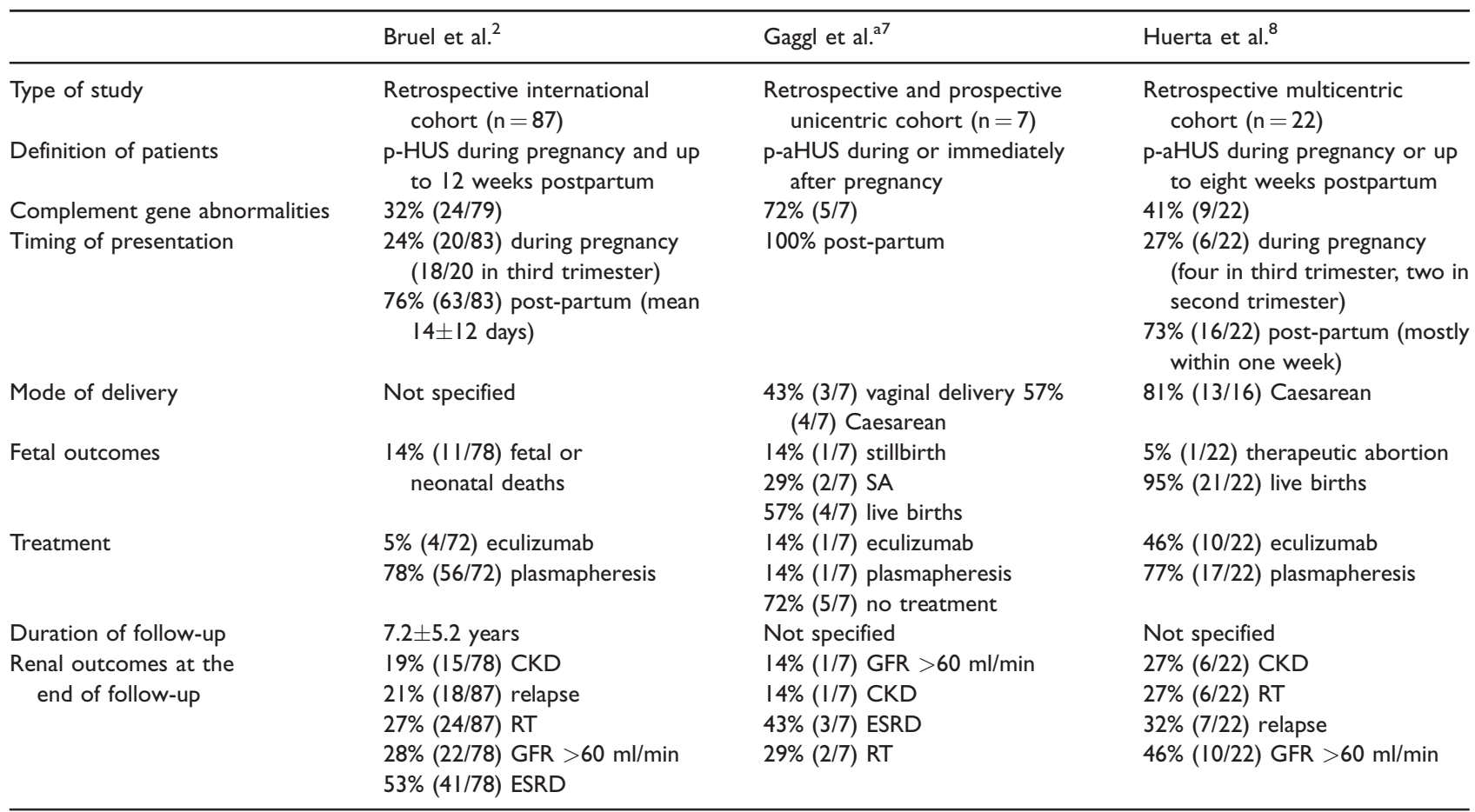

CKD: chronic kidney disease; ESRD: end-stage renal disease; GFR: glomerular filtration rate; p-aHUS: pregnancy-associated atypical hemolytic uremic syndrome; p-HUS: pregnancy-associated hemolytic uremic syndrome; RT: renal transplant; SA: spontaneous abortions.

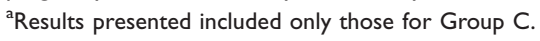

information was available on the pregnancy and delivery outcomes (mode of delivery, gestational age at delivery, etc.). Moreover, complement gene studies were not done at the same center and there is no description on the technique used for these investigations. The registries included women from 1983 to 2013, a time period during which the management of aHUS has evolved. There were limited data on the use of eculizumab. More studies are needed to assess the safety and efficacy of eculizumab in p-aHUS.

\section{Outcomes of pregnancy in women with atypical hemolytic uremic syndrome}

An Austrian TMA database was used to study maternal and fetal outcome of pregnancies in women with aHUS. ${ }^{7}$ Women were recruited from the Thrombotic Microangiopathy Cohort of the Division of Nephrology and Dialysis at the Medical University of Vienna, with a timeframe ranging from 2002 to 2015. The cases included biopsy proven or laboratory signs of TMA (mechanical hemolysis and hemoglobin $<100 \mathrm{~g} / \mathrm{L}$, platelet count $<150 \times 10^{9} / \mathrm{L}$ or drop $>25 \%$ in $48 \mathrm{~h}$, lactate dehydrogenase $(\mathrm{LDH})>250 \mathrm{U} / \mathrm{L}$, haptoglobin levels $<1.2 \mu \mathrm{mol} / \mathrm{L}$ and the presence of schistocytes in peripheral blood smear, associated with AKI and absence of ADAMTS13 deficiency or inhibitor, sequence variations within complement regulatory protein genes, and absence of secondary forms of TMA. Data collection was made by chart review. Details on genetic investigations were available in supplemental material. Outcomes were not defined.

The study included 27 pregnancies in 14 women including 9 pregnancies in 4 women that were followed prospectively. The mean age at diagnosis was $29 \pm 12$ years. They divided women into three groups: Group A included two women (five pregnancies) who were diagnosed with aHUS 6 and 14 years before their first pregnancy, Group B of five women (eight pregnancies) diagnosed with aHUS after their last pregnancy (median of 6 years, range: 2-23), and Group $\mathrm{C}$ of seven women who were diagnosed with p-aHUS during or immediately after one of their pregnancies (7/14 diagnosed during pregnancy, $71 \%$ in their first pregnancy). In the last group, triggers were identified for three of these women (infection, bleeding, and curettage after spontaneous abortion) and three women were diagnosed with preeclampsia or HELLP syndrome before the diagnosis of $\mathrm{p}$-aHUS was made. Complement gene mutations were found in $71 \%$ (10/14) of women (2/2 in-Group A, 3/5 in-Group B, 5/7 in-Group C).

Retrospective data were reviewed for 18 pregnancies and 6 were diagnosed with p-aHUS (all postpartum and all in Group C). Of those six, one recovered completely after plasmapheresis, one developed progressive chronic kidney disease without treatment and three were treated with plasmapheresis but developed ESRD. One of these received a kidney transplant. They also followed prospectively nine pregnancies in four patients (two in Group A and two in Group C). The first woman had her first episode of aHUS at three years of age, but was officially diagnosed after 22 subsequent episodes. She had two pregnancies under prophylaxis with FFP $(3 \mathrm{ml} / \mathrm{kg}$ twice a month). She developed high blood pressure with proteinuria without an increased creatinine in her second pregnancy. The second woman had received a kidney transplant for aHUS before her pregnancies. There was no complication in her first one, in which she received FFP $(2.5 \mathrm{ml} / \mathrm{kg}$ twice a month) in prophylaxis. She did not receive any treatment in her two subsequent pregnancies (no explanations given); no complications occurred in the first pregnancy, but she experienced a late in utero death related to infection in the second one. The third woman received a diagnosis of p-aHUS after her first pregnancy and 
received 26 treatments with plasmaphereses. In her two subsequent pregnancies, she received FFP $(10 \mathrm{ml} / \mathrm{kg}$ twice a month) and had no recurrence. The last woman was diagnosed with aHUS 12 months after her first pregnancy, progressed to ESRD, and received a kidney transplant. She was treated afterwards with FFP ( $25 \mathrm{ml} / \mathrm{kg}$ monthly). The frequency was increased to twice a month during her second and third pregnancies and she received supplemental infusion of FFP $(1600 \mathrm{ml})$ on the day before and after her Caesarean section. There was no complication in her second pregnancy, but she developed AKI within hours of her Caesarean section in her third pregnancy and was treated with eculizumab.

Globally, the median gestational age was 38 weeks (interquartile range (IQR) 33-40) at delivery: 70\% (19/27) of deliveries were at term, two were pre-term ( 26 and 35 weeks), two were stillborn ( 31 and 33 weeks) and four were early spontaneous abortions $(<21$ weeks). Caesarean sections were done in $65 \%$ of all deliveries after 21 weeks of gestation. In women who developed p-aHUS, there were two live term births, three preterm (two live and one stillbirth) and two early spontaneous abortions. In total, there were 21 live infants, and the rate of adverse fetal outcomes was 22 per 100 pregnancies $(95 \%$ confidence interval $(\mathrm{CI}): 10-41)$ and was 70 per 100 pregnancies (95\% CI: 33-93) for pregnancies complicated by p-aHUS. At the end of follow-up, six women still had their native kidneys, but only two of them had normal renal function, while eight women developed ESRD (seven received kidney transplant). The rate of ESRD in all 14 women was 6.36 per 100 person-years (95\% CI: 2.56-13.11).

The authors conclude that as only a quarter of their 27 studied pregnancies were complicated by episodes of p-aHUS, and as fetal outcomes were excellent in those with antenatal episodes of aHUS, outcomes of pregnancies in women with aHUS is much better than commonly reported.

This observational study is very descriptive, but there are some concerns. Groups A and C illustrated the potential impact of aHUS on pregnancy if it was diagnosed many years before, during pregnancy or early postpartum. The long-term follow-up of some of these women including nine pregnancies studied prospectively which represents a valuable contribution of this study. It may assist clinicians in the counseling of women with a personal history of aHUS regarding future pregnancy. However, the inclusion of Group B does not add any important information on the course of p-aHUS, as these women were diagnosed with aHUS at least two years after their last pregnancy. Moreover, the authors did not prospectively define their outcome measures. For example, they report rate of adverse fetal outcomes in the p-aHUS cohort, but there were no specifications on which fetal outcomes were included in this calculation. Hence, no statistical analyses can be drawn from this paper, and the conclusions are somewhat limited. More studies are needed to really assess the risk of recurrence of p-aHUS and the clinical outcomes of pregnancy after an episode of aHUS.

\section{Pregnancy-associated atypical hemolytic uremic syndrome outcomes in a Spanish cohort}

A retrospective study of the Spanish aHUS Registry evaluated epidemiological, clinical, and prognostic data of p-aHUS and the incidence of complement abnormalities. ${ }^{8}$ The authors also reported their experience with eculizumab. Data were obtained from the Database of aHUS and C3 Glomerulopathy (C3G), a registered clinical and basic research resource, funded and supervised by the Centro de Investigacion Biomédica en Red de Enfermedades Raras. Patients were identified in 13 Spanish hospitals between 1981 and 2017. Pregnancy-associated aHUS was defined as aHUS occurring during pregnancy or puerperium (up to eight weeks postpartum). Atypical HUS was defined as platelet count $<150 \times 10^{9} / \mathrm{L}$ or decrease $>25 \%$ from baseline values, hemolytic anemia, and serum creatinine level greater than the upper limit of normal range, together with a negative Coombs test, normal activity of ADAMTS13 and negative Shiga toxin. Ten patients were treated with eculizumab at a dose of $900 \mathrm{mg}$ IV per week for four weeks and then $1200 \mathrm{mg}$ IV every two weeks, and the duration was chosen by the treating physician. Plasmapheresis was discontinued when eculizumab was started. The primary outcome was the hematologic response (normalization of platelet count and disappearance of all markers of microangiopathic hemolytic anemia) and renal response (recovery of renal function with $\geq 25 \%$ reduction in serum creatinine levels). Secondary outcome was the development of ESRD, defined as the need for renal replacement therapy (RRT: dialysis or transplantation). Data collection was done through medical records.

They identified 22 patients within their registry. The median age was 33 years (73\% primiparous). None of these women had a family history of p-aHUS but $18 \%(4 / 22)$ of them had a previous episode of aHUS not related to pregnancy. More than half of the cases $(16 / 22$, $73 \%$ ) occurred in the postpartum period (mostly within the first week). Almost all of these women $(13 / 16,81 \%)$ delivered by Caesarean section and three of them with heavy bleeding. In the six women who developed p-aHUS antepartum, three were diagnosed in the 36th week of pregnancy, one in the 32nd week, one at 19 weeks who delivered five weeks later, and one who had a therapeutic abortion for hydrops fetalis also at 19 weeks. Complement abnormalities in the aHUS candidate genes were detected only in $41 \%(9 / 22)$ of the patients. Hemodialysis was started in nine women $(41 \%)$, and for the others, the maximum creatinine level was $3.5 \mathrm{mg} / \mathrm{dL}(310 \mu \mathrm{mol} / \mathrm{L})$. The minimum hemoglobin level was $6.9 \mathrm{~g} / \mathrm{dL}(69 \mathrm{~g} / \mathrm{L})$, and platelet count $50 \times 10^{9} / \mathrm{L}$. TMA was described in $10 / 11$ kidney biopsies.

Plasmapheresis was used in $77 \%(17 / 22)$ of patients for a median of six sessions. However, there were only eight hematologic and three renal responders. Almost half of the patients $(10 / 22,45 \%)$ received eculizumab at a median time of 17 days (IQR: 8-23), and all of them had hematologic and renal response. Only one received it in a context of relapse, while all the others received it after a non-response to plasmapheresis (three already required hemodialysis). Only one woman received eculizumab during pregnancy (third trimester). No secondary effects were reported and women received it ongoing treatment for a mean of 9.8 months (IQR: 7.4-12.3). All patients who received eculizumab had renal recovery, although $3 / 10$ initially required RRT. In the group who did not receive eculizumab, $50 \%$ (6/12) required hemodialysis at onset, 33\% (4/12) reached ESRD after the first month, and 50\% (6/12) still required RRT at the end of follow-up. In the entire cohort, recurrences occurred in seven patients (two after discontinuation of eculizumab), at an average of 240 months (IQR: 134.2-346.6) and one death was reported 28 years after p-aHUS.

The authors conclude that $\mathrm{p}$-aHUS is rare and is associated with complement genetic variants in $41 \%$ of patients, which was less than reported in other studies. The postpartum period seems to be the most at-risk period and their results suggest an association with Caesarean section, related to the higher endothelial damage and heavier bleeding. Finally, the use of eculizumab was safe and demonstrated higher efficacy than plasmapheresis in terms of hematologic and renal response.

This retrospective Spanish database cohort presents many interesting results. Although the number of included women was small (22), the definitions of cases and outcomes were clear and allow us to draw some valuable conclusions. The postpartum period was related to a higher risk of p-aHUS and this risk seems to be greater following Caesarean section. Another strength of this study is the data on the use of eculizumab in 10 women. It is the highest reported number 
of women treated with eculizumab for p-aHUS. Renal and hematologic responses were achieved and no side effects were reported. However, only one woman received eculizumab during pregnancy. Thus, more studies are needed to assess safety of eculizumab in paHUS during pregnancy.

\section{Conclusion}

p-aHUS is a very rare disease with potentially catastrophic consequences. Clinicians should be aware of this syndrome and suspect it in the presence of TMA associated with thrombocytopenia and AKI. Complement gene abnormalities studies can help confirm the diagnosis but may take many weeks to be completed, thus they might not be useful in the acute situation. If there is suspicion of p-AHS, treatment with eculizumab should be considered, as it improves renal and hematologic prognosis. However, as eculizumab is expensive and less readily available in different resource settings, plasma exchange should be started as soon as possible as bridging therapy.

\section{Acknowledgements}

The authors thank Ghada Bourjeily for assistance to this study.

\section{Declaration of conflicting interests}

The author(s) declared no potential conflicts of interest with respect to the research, authorship, and/or publication of this article.

\section{Funding}

The author(s) received no financial support for the research, authorship, and/or publication of this article.

\section{Ethical approval}

Not applicable.

\section{Guarantor}

SGM.

\section{Contributorship}

SGM wrote the manuscript and SL reviewed and edited the manuscript.

\section{References}

1. Jokiranta TS. HUS and atypical HUS. Blood 2017; 129: 2847-2856.

2. Bruel A, Kavanagh D, Noris M, et al. Hemolytic uremic syndrome in pregnancy and postpartum. Clin J Am Soc Nephrol 2017; 12: 1237-1247.

3. Shatzel JJ and Taylor JA. Syndromes of thrombotic microangiopathy. Med Clin North Am 2017; 101: 395-415.

4. Fakhouri F. Pregnancy-related thrombotic microangiopathies: clues from complement biology. Transfus Apher Sci 2016; 54: 199-202.

5. Dashe JS, Ramin SM and Cunningham FG. The long-term consequences of thrombotic microangiopathy (thrombotic thrombocytopenic purpura and hemolytic uremic syndrome) in pregnancy. Obstet Gynecol 1998; 91: 662-668.

6. Legendre CM, Licht C, Muus P, et al. Terminal complement inhibitor eculizumab in atypical hemolytic-uremic syndrome. $N$ Engl J Med 2013; 368: 2169-2181.

7. Gaggl M, Aigner C, Csuka D, et al. Maternal and fetal outcomes of pregnancies in women with atypical hemolytic uremic syndrome. J Am Soc Nephrol 2018; 29: 1020-1029.

8. Huerta A, Arjona E, Portoles J, et al. A retrospective study of pregnancy-associated atypical hemolytic uremic syndrome. Kidney Int 2018; 93: 450-459. 